Brit. Heart F., 1967, 29, 641.

\title{
Surgery of the Mitral Valve
}

\author{
RICHARD W. D. TURNER \\ From the Department of Medicine, University of Edinburgh
}

Whereas previously the myocardial factor was considered dominant, the introduction of surgical treatment for valvular disease and its successful practice have established the importance of valvular obstruction and incompetence in the production of symptoms whether or not significant myocardial damage is also present. Other factors, of course, including secondary changes in the pulmonary vessels and lungs and the effects of congestive failure, particularly on the liver, can influence long-term results and the operative risk. Embolism from displacement of clot is an ever present hazard.

The commonest valvular defect is mitral stenosis with varying degrees of sclerosis and calcification of the cusps but little or no associated incompetence, or aortic valvular disease other than mild incompetence. In some cases of mitral stenosis there is significant but still relatively unimportant incompetence. In others stenosis and incompetence are of approximately equal hæmodynamic significance as far as can be judged from clinical examination, radiography, electrocardiography, catheterization studies and angiocardiography. In addition there may be any degree of associated aortic or tricuspid valvular disease. Pure or dominant incompetence is present more frequently than used to be realized.

The myocardial factor is always important. There may be relatively simple and largely reversible hypertrophy, past rheumatic myocardial damage or active carditis. Myocardial damage may be difficult to assess but is largely reflected in individual chamber enlargement from dilatation rather than hypertrophy, and in a low cardiac output when this is not attributable to a raised pulmonary vascular resistance.

Atrial fibrillation is probably most often related to myocardial damage but there is not always a good correlation. Clot is more likely to be present than with sinus rhythm.

Increasing age brings with it an increasing incidence of calcification of the valve and other adverse factors associated with rheumatic heart disease and also coronary arterial disease, systemic hypertension, pulmonary disease, and other disorders.

There is a changing pattern in the age and grade of disease among those coming to operation and in the severity of rheumatic heart disease. There are now fewer young persons with rheumatic heart disease and fewer with uncomplicated mitral stenosis. Probably the majority of those undergoing surgical treatment for rheumatic heart disease in this country are older and have a relatively complex disorder for which previously nothing could be done. Since the introduction of the bypass technique, a new group of patients can be offered treatment.

Surgical treatment should be advised, irrespective of the presence or absence of symptoms, in all patients who have severe stenosis. Justification for this view lies in experience of the adverse effects of delaying operation in those who at the time of surgical treatment clearly have long-standing stenosis. In a study in London some years ago a group of patients, with significant mitral stenosis, who were not operated on, was followed, with disastrous results, mainly in the form of systemic embolism. It is evident that this group of patients had a greater degree of stenosis than was appreciated.

In the majority of patients with the physical signs of uncomplicated mitral stenosis and undue breathlessness on exertion, not attributable to some other cause, the degree of stenosis is sufficient to warrant surgical treatment. In a few there may be a low threshold for dyspncea as for pain but in the great majority of cases the rule holds true. Undue breathlessness may be due in part to other factors such as anæmia, or respiratory disease. Some patients deny relevant symptoms though there is unequivocal evidence of severe stenosis on physical examination, radiography, electrocardiography, or cardiac catheterization.

There are few absolute contraindications to the relief of tight stenosis. Relative contraindications include atrial fibrillation, age over 50 , heavy calcifi- 
cation of the valve, a large heart, associated mitral incompetence, aortic stenosis or incompetence, tricuspid stenosis or incompetence, and a high pulmonary vascular resistance, but none of these or usually any combination of them should preclude the relief of important obstruction. There are of course occasional exceptions. Inoperable carcinoma might be regarded as a contraindication, also severe hemiplegia in the absence of dyspnœea, but not if there is evidence of progressive cardiac hypertrophy or enlargement or if breathlessness at rest develops, so long as the patient is mentally robust. Aortic or tricuspid valvotomy can when necessary be carried out at the same thoracotomy.

It is not always appreciated that severe mitral stenosis may be present despite a loud apical systolic murmur. It is usually not difficult to distinguish an apical systolic murmur due to aortic stenosis, but despite theoretical considerations of timing and quality it is not always easy to be certain that aortic stenosis and mitral incompetence are not both present, and a left ventricular angiogram may be necessary.

A much more frequent error is failure to appreciate that a loud apical systolic murmur may be due to tricuspid incompetence with clockwise rotation of the heart as viewed from the apex. In such cases there is always evidence of severe pulmonary hypertension with right ventricular hypertrophy, and usually the murmur disappears or is greatly reduced after mitral valvotomy. In the context of mitral incompetence it is also important to appreciate that a central systolic lift is not always due to right ventricular hypertrophy but may result from dilatation of the left atrium with ventricular systole.

There are many traps for the unwary, but attention to detail and the utilization of modern methods of investigation in properly equipped centres minimize the dangers of a mistake.

The first prerequisite for successful surgical treatment is of course accurate diagnosis, but if on other grounds, such as heavy calcification recognized at a previous valvotomy, or the need to replace the aortic or tricuspid valves, it has been decided that openheart surgery is necessary, precision as regards the relative degrees of mitral stenosis and incompetence may now be less important.

Nevertheless a frequent problem is to decide whether the degree of associated incompetence is sufficient to preclude a closed transventricular valvotomy, and in such cases cardiac catheterization and left ventricular angiocardiography should be carried out.

In many patients with signs of uncomplicated mitral stenosis catheterization is unnecessary, especially if there is radiographic evidence of pulmonary œdema or pulmonary arterial hypertension or an electrocardiographic pattern of right ventricular hypertrophy. In the absence of incompetence the valve area can be calculated if the gradient across it in diastole and the cardiac output are known. If the mean left atrial pressure is high some form of surgical treatment is indicated. The left atrial pressure pulse is an unreliable guide to the severity of incompetence because it is also dependent on the pressure volume characteristics of the atrium, which in many cases are mainly determined by rheumatic myocardial damage. In some cases of incompetence there may be a considerable gradient across the valve in diastole. Calculation of the rate of descent of the $v$ wave ( $y$ descent) by the formula devised by Owen and Wood $(\mathrm{Ry} / \mathrm{v})$ is unreliable. Dye dilution techniques for computation of mitral incompetence in the individual patient have not proved reliable partly due to errors of sampling and also because cardiac output and left atrial volume are important factors. Left ventricular cine-angiography in centres where adequate experience has been gained is an approximate guide to incompetence but quantitation is not accurate. Nevertheless by a combination of clinical, radiographic, electrocardiographic, hæmodynamic, and dye dilution techniques considerable accuracy can be achieved. In many cases no special investigations are required other than radiography and electrocardiography.

In a few centres all patients with mitral stenosis are treated under direct vision with cardiac bypass. It is claimed that the operative risk is little greater than for closed operations, a more satisfactory valvotomy can be achieved, associated incompetence can if necessary be repaired, and the valve can be replaced when indicated. However, in most centres closed valvotomy is still advised at least for a first operation, because the operative risk is lower and long-term results are satisfactory in the majority of cases for a considerable number of years. In addition, as discussed below, there are valve replacement disadvantages in the form of embolism, sepsis, detachment of the prosthesis, obstruction to the left ventricle, and the unknown durability of the materials.

In patients with heavily calcified valves in particular it is now sometimes considered that valve replacement should always be carried out at a first operation, but there are frequently important associated adverse factors which increase the operative risk not only for closed procedures but for valve replacement, and again, in the majority of those who survive the operation the long-term results after valvotomy are reasonably satisfactory for several years.

The fact is that the safety of open-heart surgery, 
the design of prostheses, and the materials used are likely to continue to improve for some time, so that for many patients it is best to have a closed first valvotomy in the knowledge, of the medical advisers at least, that by the time a second operation may be required the situation will have considerably improved.

Open-heart surgery is advisable if there is significant mitral incompetence or if at a previous closed operation the valve was found to be heavily sclerosed or calcified.

In Edinburgh it is still our practice to advise transventricular valvotomy as a first procedure in almost all patients with pure or dominant mitral stenosis.

\section{OPERATIVE MORTALITY}

The operative risk in the individual patient cannot be assessed from the over-all mortality in a large series of patients with varying degrees of mitral stenosis or incompetence, calcification of the valve, associated aortic and tricuspid disease, and secondary changes in the pulmonary circulation and liver. Numerous factors must be taken into account in each case. The risk is low in the relatively young in sinus rhythm and without much cardiac enlargement, increased pulmonary vascular resistance, or calcification of the valve, and relatively high in the older age-groups with atrial fibrillation, large hearts, damaged lungs, and heavy calcification of the valve.

\section{Operative Complications}

The principal and unpredictable hazards of valvotomy are systemic embolism and traumatic mitral incompetence. The incidence of embolism should now be low, and there is no convincing evidence that it can be reduced by the previous administration of anticoagulants. Possibly the main factors in the lower incidence in any centre are increasing experience and improved techniques. The incidence of traumatic mitral incompetence varies considerably between centres, but with the proper use of the transventricular dilator it should be low.

In some centres it has been found that, with the transventricular dilator, better separation of the cusps can be obtained in poorer valves though the incidence of traumatic incompetence is greater. This has not been our experience, owing probably to the correct use of this instrument which permits the controlled use of force, the blades being opened millimetre by millimetre with palpation at each stage to detect the earliest evidence of regurgitation. Should incompetence be produced facilities for repair with the bypass technique should be available.

\section{LONG-TERM RESULTS}

The long-term results of valvotomy are surprisingly good when it is remembered that surgical treatment does not affect the damaged myocardium, except in so far as the hæmodynamic burden is reduced, nor does it affect the relentless progression of the sclerosis which so often follows initial infection or recrudescence. However, in most cases the need for another operation increases with time. It has not been established that the necessity for a second operation is closely related to the extent of the first valvotomy.

There is a relatively small group of patients in whom, after successful valvotomy, subjective and objective evidence of improvement continues for 15 years or more, with no evidence of deterioration or any change in physical signs.

The ætiology of mitral incompetence can usually be presumed from the history. In the relatively uncommon cases of acute incompetence there may be a history of trauma, myocardial infarction, or bacterial endocarditis but ruptured chordæ can occur in otherwise normal valves.

In the usual case, with or without a past history of rheumatic fever, there is a gradual onset of symptoms and either evidence of some degree of associated mitral stenosis or other valvular defects or the characteristic radiographic appearances of dilatation of the auricle and atrium, and in the great majority of cases it is safe to assume a rheumatic origin. A congenital form of mitral incompetence may be present with a cleft anterior cusp of the valve due to maldevelopment of the endocardial cushions and partial or total persistence of the atrio-ventricular canal.

From clinical examination, radiography, and electrocardiography, it is not always possible to be certain whether mitral incompetence alone or in association with other valvular defects is of sufficient degree to warrant surgical treatment. The problem is not difficult if, in addition to a loud apical systolic murmur well heard towards the axilla and left lung base, there is a third heart sound, and clinical, radiographic, or electrocardiographic evidence of ventricular enlargement or hypertrophy. In addition there may or may not be a short mitral diastolic murmur from increased blood flow. However with all valvular defects the loudness of a murmur does not in the individual patient necessarily bear any close relation to the severity of incompetence or obstruction, and reliance should never be placed upon this.

A third heart sound is usually reliable evidence of severe incompetence. However, since in any form of cardiomyopathy a third sound may occur during rapid ventricular filling without valvular 
incompetence it might on occasion be misleading. This possibility is also suggested by the fact that despite successful repair of the valve, with disappearance of the apical systolic murmur a third heart sound may persist.

With mitral incompetence in particular surgical treatment should not wait upon symptoms but should depend on objective evidence of severity. If the evidence is only angiographic, it is probably wiser at the present time to wait, because in most centres techniques and, with greater experience, the safety of operation are improving. Nevertheless, in the "good risk" group the operative risk is now low, and it is better to advise surgical treatment if there are signs of progressive left ventricular enlargement or hypertrophy and before there is any evidence of left ventricular failure. It is worth emphasizing that symptoms should not be attributed to mitral incompetence unless there is evidence of left ventricular failure and that the onset of symptoms reflects an advanced stage of the disease.

Where repair of the valve is possible this is much to be preferred to replacement, despite the fact that it cannot be predicted whether, after apparently successful repair, incompetence will return through partial cutting through of sutures. This has occasionally happened but on the other hand it may well be that these cases were unsuitable for repair in that there was not sufficient thickening of the tissues for sutures to hold. If the incompetent valve is very sclerotic or calcified, replacement is advisable because these pathological changes are usually progressive.

The advantages of repair lie in the avoidance of introducing foreign material other than the sutures. The disadvantages of valve replacement with the prostheses at present available include embolism, sepsis, detachment, and the fact that in all cases the prosthesis produces some degree of stenosis and reduction of left ventricular volume. This last factor is much more likely to affect patients with mitral stenosis because in the majority of those with significant incompetence, the ventricle is dilated as well as hypertrophied.

There are a number of causes for mitral incompetence and the best procedure to be carried out will be determined by the nature of the anatomical defect. In most cases incompetence is the consequence of rheumatic fever which causes fibrosis of the cusps with shrinkage of the chordæ and sometimes, in varying degree, dilatation of the valve ring. Incompetence solely due to isolated dilatation of the ring with normal cusps is rare. When incompetence is the result of bacterial endocarditis there may be loss of tissue. In these cases the valve should be replaced.
The operative risk is mainly related to the extent of myocardial damage by the rheumatic process with or without other valvular defects and secondary changes in the lungs and liver. Atrial fibrillation is usually an indication of myocardial damage, but, surprisingly, some patients with apparently severe myocardial damage maintain sinus rhythm whereas others develop atrial fibrillation at a relatively early stage.

Left ventricular failure results in pulmonary venous congestion and a low cardiac output. Peripheral signs of congestive failure represent an even more advanced stage of the disease, but this is not in itself a contraindication to surgical treatment and a remarkably good result may follow.

The defect usually seen at operations for mitral incompetence is a rise of the middle third or more of the free margin of the aortic cusp into the atrium above the mural cusp during ventricular systole. This has been seen with pure incompetence and with dominant incompetence with some stenosis, with a wide ring and with a narrow or normal ring, with a broad mobile mural cusp, with a shrunken fixed mural cusp and with intact chordæ.

The procedures most commonly used for correction of mitral incompetence are plication of the annulus and replacement of the valve. Plication is indicated where there is only moderate sclerosis of the valve without calcification and with no more than minor stenosis which can be reduced by valvotomy at the same time. The annulus is plicated by simple sutures of terylene 1-4 in number anteriorly and posteriorly in relation to the commissures. The cusps themselves are not involved in the sutures and because of the natural funnel shape of the mitral valve the annulus can be narrowed with little or no diminution of the valve orifice. No suture encroaches on that part of the valve which is continuous with the root of the aorta; that is, all sutures are on the mural side of the line continuing the crescentic slit of the valve to the ring. Replacement of the valve is necessary when its structures are heavily sclerosed, distorted, or destroyed so that valve function cannot be restored by conservative means. In some cases in which the cusps are shrunken but mobile and the chordæ are of normal length, enlargement of one or other cusp by the introduction of a gusset of pericardium may seem preferable to plication. When a cusp has been torn at a previous blind valvotomy incompetence may be overcome by simple suture of the tear in the cusp. However, a cusp is unlikely to have been torn unless the valve was heavily sclerosed, so that in this group replacement is usually required.

The incompetence caused by chordal rupture can sometimes be overcome by simple plication of the 
flail part of the cusp. When this is unsuccessful replacement is likely to be necessary.

As with mitral valvotomy the operative risk depends on a number of variable factors and many patients treated by valve repair or replacement have severely damaged hearts. Among my own patients in the past two years or so operative mortality has been approximately 10 per cent for repair and 15 per cent for replacement. However, the disease in many of these patients was in an advanced state and treatment is now being offered earlier with a considerably lower mortality.

The incidence of subsequent embolism has not been high, amounting to three cases in each group. In two, with valve replacement, embolism occurred in relation to sepsis round the prosthesis. In three of the remaining four patients the adequacy of anticoagulant control was very doubtful.

Sometimes after mitral valve replacement there is considerable reduction in heart size but in others little or none, and in a few heart size as judged by the cardiothoracic ratio is increased. In the Edinburgh series incompetence round the prosthesis has been rare so that this cannot be the explanation. It is possible that in some patients with a relatively small ventricle there is a significant degree of obstruction by the prosthesis and left ventricular performance may be impaired by absence of the chordæ tendinex. In many patients there has been rheumatic myocardial damage before operation.

It is noteworthy that heart size seems to decrease more after aortic valve replacement. All these factors require further study.

I should like to thank my surgical colleague, Andrew Logan, who has carried out most of the operations, and my medical colleague, Arthur Kitchin, who is in charge of the cardiac laboratory, for their advice in the preparation of this editorial article. 